\title{
CEREBELLAR PURKINJE CELLS ARE DESCENDED FROM A SMALL NUMBER OF PROGENITORS COMMITTED DURING EARLY DEVELOPMENT: QUANTITATIVE ANALYSIS OF LURCHER CHIMERIC MICE ${ }^{1}$
}

\author{
RICHARD WETTS ${ }^{2}$ AND KARL HERRUP
}

Department of Human Genetics, Yale University School of Medicine, New Haven, Connecticut 06510

Received December 7, 1981; Revised April 5, 1982; Accepted May 13, 1982

\begin{abstract}
Previous analysis of lurcher $\leftrightarrow$ wild type aggregation chimeras revealed that the degeneration of cerebellar Purkinje cells (PCs) in lurcher mutants is due to a defect intrinsic to the PCs themselves. Thus, all of the PCs remaining in lurcher chimeric mice are descended from only the wild type embryo. In this study, we have determined the number of PCs in four lurcher chimeras and three wild type mice.The low number of wild type PCs $(10,200)$ in half of the cerebellum of one chimera $(\chi 11)$ suggested that these cells might be descended from a single progenitor. This idea is strongly supported by the quantitative analysis of the PCs in the other animals. These numbers are integral multiples of the number of PCs in $\chi 11$. We believe that each stepwise increase in the number of PCs in the chimeras is due to the addition of a single wild type cell to the progenitor pool. The existence of integral multiples implies that no other cells can contribute to the PC population after the progenitors become commilted to forming PCs. We cannot know, however, whether the PC progenitors also give rise to other cell populations. The values of the integrals in wild type mice indicate that the entire $\mathrm{PC}$ population descends from a small number of progenitors $(8 \mathrm{in} \mathrm{C} 3 \mathrm{H} / \mathrm{HeJ}$ mice). We calculate that these progenitors are committed during the neural plate to neural fold stage of development. Thus, the fate of the progenitors of the cerebellar PCs is restricted very early in neural development.
\end{abstract}

A major challenge of developmental neurobiology is to determine the stage when a neuron's fate becomes established. While overt differentiation usually does not occur until late in development, the particular genetic program that a cell will employ can be selected much earlier. The fate of neural crest cells has been shown to be determined during and after migration from the neural tube. The decision to differentiate into a specific cell type is based on cues from the cell's immediate surroundings (LeDouarin, 1980). Examples from central nervous system structures suggest that commitment can precede the final cell division as is the case for neurons in the lateral motor

\footnotetext{
${ }^{1}$ We would like to thank Mr. Thomas Diglio and Ms. Sandra Wilczynski for their technical assistance. This work was supported by a Basil O'Connor Starter Grant from the March of Dimes, by Grant HD 12213 from the National Institutes of Health (K. H.), and by National Research Service Award HD07149 from the National Institutes of Health (R. W.). This work is in partial fulfillment of the requirements for the degree of Doctor of Philosophy at Yale University (R. W.).

${ }^{2}$ To whom correspondence should be addressed at Department of Human Genetics, Yale University School of Medicine, 333 Cedar Street, New Haven, CT 06510.
}

column (Wenger, 1951) and for the radial glial cells (Levitt et al., 1981). The work reported here addresses this question for the cerebellar Purkinje cells of the mouse.

We have performed a quantitative cell lineage analysis of mutant $\leftrightarrow$ wild type chimeric mice. A chimera is formed by aggregating two embryos with different genotypes, thereby forming a single embryo composed of mutant and wild type cells (McLaren, 1976; Mintz, 1974). The nervous system of the adult chimera is a fine grained mosaic of mutant and wild type cells. For our analysis, we used the lurcher mutation (gene symbol, $L c$ ). Lurcher is an autosomal dominant mutation (Phillips, 1960) in which the heterozygotes lose $100 \%$ of their Purkinje cells (PCs) during early postnatal life (Caddy and Biscoe, 1979). In lurcher $\leftrightarrow$ wild type chimeras, there is a reduced number of PCs. Using $\beta$-glucuronidase activity as an indepedent cell marker (see the description in Mullen and Herrup, 1979), all of the PCs remaining in the chimeras were identified as wild type cells. All of the $+/ L c$ cells had degenerated. The correlation between phenotype (presence or absence) and genotype (wild type or lurcher) indicates that the degeneration of the PCs is due 
TABLE I

Corrected number of Purkinje cells in half of the cerebella of four chimeras and three control mice

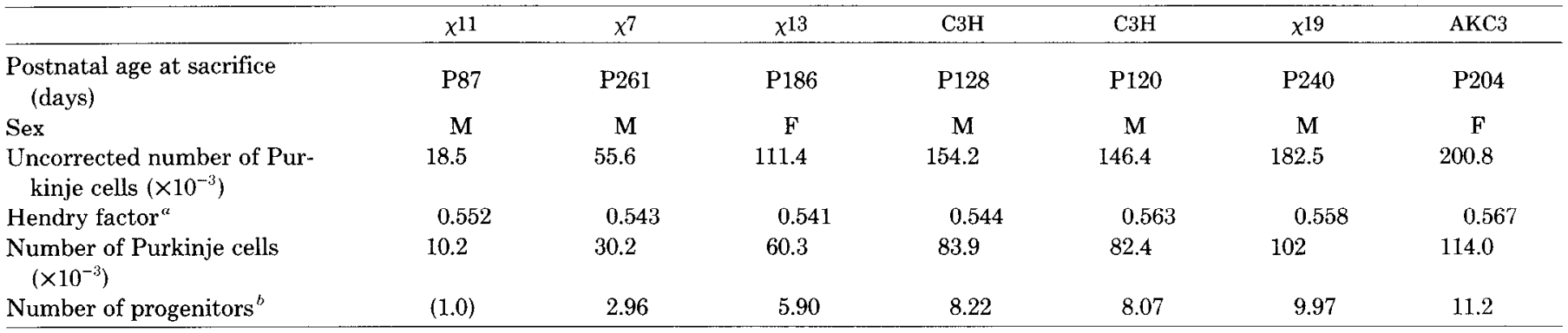

a This number is a reflection of the extent to which the uncorrected number of Purkinje cells is an overestimate of the true number. It is calculated according to the equations of Hendry (1976).

${ }^{b}$ The number of Purkinje cells in each animal was divided by the number of Purkinje cells in $\chi 11$ to obtain the number of progenitors. These values are close to integers, which indicates that the progenitors (and no other cells) gave rise to the entire population of Purkinje cells. In the chimeras, any other progenitors were $+/ L c$ in genotype, and thus, their descendants had degenerated. The value for $\chi 11$ is enclosed in parentheses to indicate the fact that it is arbitrarily set equal to 1.0 .

to an intrinsic defect (Wetts and Herrup, 1982). Thus, in $+/ L c \leftrightarrow+/+$ chimeras, only $+/+\mathrm{PCs}$ remain in the adult.

We have determined the total number of PCs remaining in four lurcher chimeras and three wild type mice. For this quantitative analysis, $L c$ is a more accurate cell marker than $\beta$-glucuronidase activity. Our data reveal the existence of numerical units of PCs. These findings suggest that a small number of progenitors gives rise to the entire population of cerebellar PCs. Furthermore, these progenitors are committed to becoming PCs very early in neural development.

\section{Materials and Methods}

Chimeras were produced by the embryo aggregation method of Mintz $(1962,1965)$ and Tarkowski (1961) with modifications (Mullen and Whitten, 1971). For each aggregation, one embryo was obtained by mating a C57BL/ $6 \mathrm{~J}$ female to $\mathrm{a}+/ L c$ male. These males were not inbred, but the $L c$ gene was being transferred to the $\mathrm{C} 57 \mathrm{BL} / 6 \mathrm{~J}$ background. The other embryo was either a $\mathrm{C} 3 \mathrm{H} / \mathrm{HeJ}$ (for $\chi 11, \chi 7$, and $\chi 13)$ or a $(\mathrm{AKR} / \mathrm{J} \times \mathrm{C} 3 \mathrm{H} / \mathrm{HeJ}) \mathrm{F}_{4}($ for $\chi 19)$. These latter strains were chosen because they differ from $\mathrm{C} 57 \mathrm{BL} / 6 \mathrm{~J}$ at the $\beta$-glucuronidase locus. This difference was needed to establish the site of $L c$ gene action, but it is not critical to the present analysis. On the 2nd day after finding a vaginal plug, the embryos were flushed from the females' oviducts. The embryos were at the 8cell stage of development and were surrounded by zonae pellucidae. The zonae were removed with Pronase, and the embryos were rinsed three times in medium. One embryo of each genotype was placed in a drop of medium under paraffin oil. Mouse embryos are naturally sticky at this stage and thus adhere to one another when they are placed in contact. The embryos were cultured overnight at $37^{\circ} \mathrm{C}$ in an atmosphere of $5 \% \mathrm{CO}_{2}, 5 \% \mathrm{O}_{2}, 90 \%$ $\mathrm{N}_{2}$. By the following day, each pair of embryos had formed a single, double sized morula. The chimeric morulae were transplanted surgically into the uteri of females made pseudopregnant by mating them to vasectomized males 2 days earlier. The chimeric embryos went to term and were born normal in size.

All of the animals were perfused transcardially, and the brains were prepared for $\beta$-glucuronidase histochemistry as described by Mullen (1977). The cerebella were sectioned serially at $8 \mu \mathrm{m}$ in the sagittal plane, and every 5 th section was stained with cresyl violet. Purkinje cells were counted in every 20 th section. In each section, every cell was counted that $(a)$ was in the PC layer, $(b)$ had a large soma, and $(c)$ had at least a portion of its nucleus in the section. All of the counting was done by the same person (R.W.), and duplicate counts of the same section were within $3 \%$ (Konigsmark, 1970). The number of PCs in each section was graphed as a function of the distance away from the midline (Fig. 1). The area under this curve is proportional to the total number of PCs in half of the cerebellum. These raw values are overestimates because cell nuclei can be split during sectioning and thus may appear in more than 1 section. The corrected number of PCs was obtained by multiplying the raw values by the Hendry (1976) correction factor for each animal (Table I). The Hendry method is based on the correction of a distribution of cell diameters to reflect only those cells whose true center appears in the section. The distribution of cell diameters was obtained by measuring the maximum diameters of all PC nuclei in a given section using a drawing tube and a digitized $\mathrm{X}-\mathrm{Y}$ tablet (Zeiss MOP$3)$.

\section{Results}

The corrected numbers of PCs for four chimeras and three wild type animals are presented in Table I. Note that one chimera, designated $\chi 11$, possessed only 10,200 PCs in the left half of its cerebellum. In our experience, we have examined qualitatively the cerebella of more than 150 mutant and control chimeras, and $\chi 11$ struck us as having among the smallest number of $\mathrm{PCs}$ of one genotype that we have ever encountered in a mosaic PC layer. The only animals with fewer PCs in this study were two nonchimeric $+/ L c$ animals which were born after transplanting chimeric embryos. ${ }^{3}$ According to clas-

\footnotetext{
Due to the nature of early mouse embryogenesis and the method of chimera production, a certain proportion of chimeric embryos can be expected to produce nonchimeric animals (Falconer and Avery, 1978). Twice during development, when the inner cell mass differentiates from the trophectoderm and when the primary ectoderm differentiates from the primary endoderm, cells are partitioned into embryonic and extra-embryonic tissues. If all of the cells partitioned into the embryonic component are the same genotype, then the resulting mouse will be nonchimeric.
} 
sical analysis (Mintz, 1974; McLaren, 1976; Dewey et al., 1976), the smallest unit of cells of one genotype seen in a chimera represents one clone. A clone is defined as a group of cells which are all descendants of a single cell. If the number of PCs in $\chi 11$ is, in fact, the smallest number possible, then these $10,200 \mathrm{PCs}$ can be taken to be a clone of cells descended from a single $\mathrm{C} 3 \mathrm{H}$ progenitor. All of the other cells which were committed to becoming PCs were $+/ L c$ in genotype. The PCs derived from these cells had all degenerated due to the intrinsic action of the $L c$ gene (Welts and Herrup, 1982).

The hypothesis that the PCs in $\chi^{11}$ represented a single clone is strongly supported by the number of PCs in the other chimeras and the wild type controls. When the number of PCs in each of these six animals is divided by the number of PCs in $\chi 11$, the quotients are not significantly different (within the $3 \%$ counting error) from integral values (Table $\mathrm{I}$ ). The $3 \%$ counting error is generally considered acceptable (Konigsmark, 1970), but we were surprised that the variance due to environmental factors, developmental processes, etc. did not distort the unit size of the PCs. Thus, the quotients for most of the animals are well within $3 \%$ of an integral multiple, and only one (the P128 C3H) comes close to, but does not

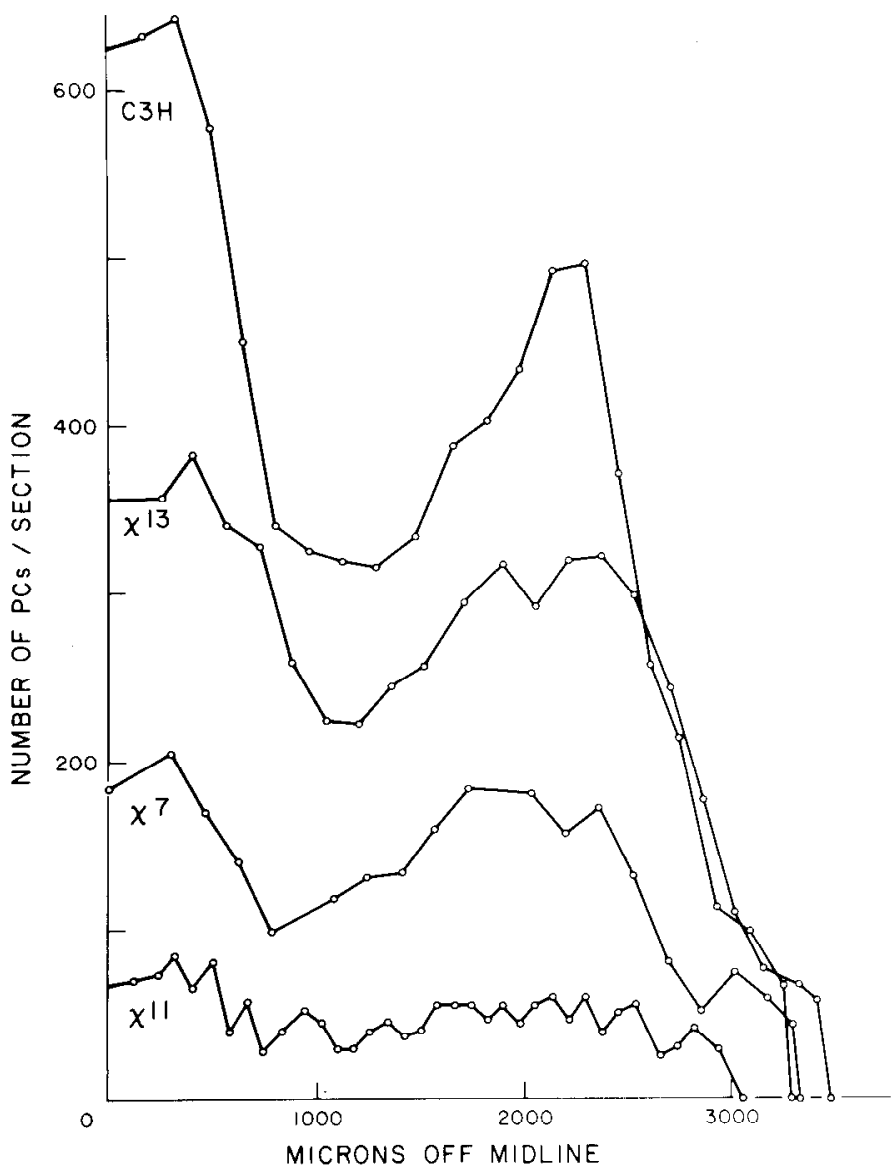

Figure 1. The number of Purkinje cells per section graphed as a function of the distance of each section from the midline. Only four of the seven animals are shown here $\left(\chi 11, \chi^{7}, \chi^{13}\right.$, and the $\mathrm{P} 128 \mathrm{C} 3 \mathrm{H}$ ). The area under each curve is proportional to the uncorrected number of Purkinje cells in half of the cerebellum. Note that, for all of the chimeras, Purkinje cells are present throughout the mediolateral extent of the cerebellum. exceed, the $3 \%$ limit. The probability that these quotients fell within $3 \%$ of an integer by chance alone is very small. Assume that the number of PCs in a chimera could vary in steps of 1 cell instead of units of 10,200 cells. Then, there would be 117,000 different possible counts of PCs (any value from 0 to $3 \%$ greater than our highest count). It can be calculated that approximately 40,400 (or $35 \%$ ) of these 117,000 values fall within $3 \%$ of an integral multiple of $\chi 11$. The likelihood that three other chimeras plus two inbred strains ( $\mathrm{C} 3 \mathrm{H}$ and the AKC3 mice) all fell within this range by chance is $(0.35)^{5}=0.0053$. While there are surely other methods of estimating these odds, this analysis suggests that we are not likely to have obtained our findings by chance alone.

In the chimeras, the integral multiples suggest that each stepwise increase in the number of PCs is due to the addition of a single $\mathrm{C} 3 \mathrm{H}$ cell to the precursor pool. Since the numbers of PCs in the nonchimeric controls are also integral multiples of the number in $\chi 11$, the existence of these integrals cannot be an artifact of the chimerism or of the $L c$ gene. The values of the integrals in the wild type animals indicate that the total number of cells which become committed is rather small (8 in $\mathrm{C} 3 \mathrm{H} / \mathrm{HeJ}$ mice) and that no other cells can join the PC progenitor pool after the commitment event occurs. However, these data do not indicate whether the PC progenitors also give rise to other cell types.

Although $\chi 11$ had only $12 \%$ as many PCs as the $\mathrm{C} 3 \mathrm{H}$ controls, neither this animal nor any other chimera displayed any marked behavioral abnormalities. In all of the chimeras, including $\chi 11, \mathrm{PCs}$ were present throughout the mediolateral (Fig. 1) and the rostrocaudal (Fig. 2) extent of the cerebellum. It seems, therefore, that the commitment event did not restrict the functional fate or anatomical location of the PCs. The descendants of each progenitor cell were capable of contributing to all functional subdivisions of the cerebellum.

\section{Discussion}

We have determined the numbers of PCs present in four lurcher chimeras and three wild type mice. In the mosaic brains of the lurcher chimeras, only the PCs descended from one of the two original embryos survive. The lurcher gene merely serves as a cell marker (analogous to an enzyme variant), allowing us to count only PCs descended from the wild type embryo. When the numbers of these PCs are divided by 10,200 (the number of PCs in $\chi 11$ ), all of the quotients are approximately integral values. What is the probability that this occurred by chance alone? Certainly, it would be desirable to have a large number of animals in order to do a rigorous statistical analysis. Unfortunately, it is difficult to produce the chimeras and tedious to count the PCs accurately. However, the calculation described above should give an adequate picture of the probability of obtaining these seven integers by chance. This probability is extremely small. A second question is whether the PCs in $\chi 11$ are descended from only 1 or from 2 progenitors. If the latter were true, the number of progenitors in the other mice would have to be doubled. In this situation, all of the quotients would be multiples of 2 , and the odds of this happening are also very low $\left(p=0.5^{6}=0.016\right)$. Thus, it is most likely that the PCs in $\chi 11$ were descended 


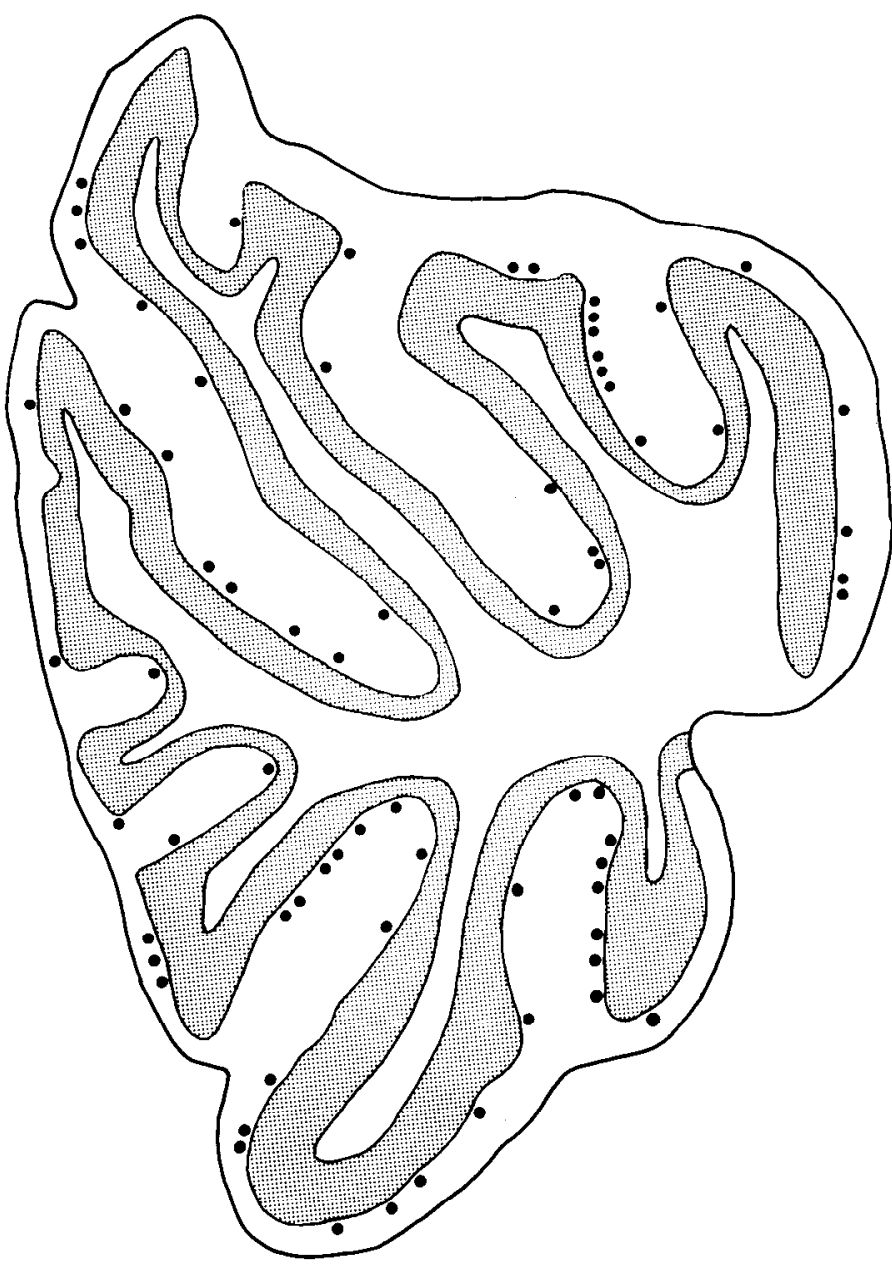

Figure 2. Location of Purkinje cells in the cerebellum of $\chi 11$. This section, $240 \mu \mathrm{m}$ from the midline, has 70 Purkinje cells (indicated by dots). A comparable section from a $\mathrm{C} 3 \mathrm{H}$ mouse has approximately 600 Purkinje cells. Note that Purkinje cells are located in every lobule. This implies that the descendants of each progenitor can contribute to all functional subdivisions of the cerebellum. Anterior is at the top and dorsal is on the left. Magnification $\times 45$.

from only 1 progenitor. Finally, if the number of $\mathrm{PC}$ progenitors were small, it would be predicted that, occasionally, all of the $\mathrm{PC}$ progenitors in a given chimera would be of one genotype while the rest of the CNS was mosaic. It is significant that an animal of this type was described, though not commented on, by Dewey et al. (1976).

The data here suggest that the entire population of $\mathrm{PCs}$ in normal mice descends from a relatively small number of progenitors. A cell lineage analysis of the contiguous patches of photoreceptors in the neural retina led Mintz to similar conclusions. A small number of progenitors, 10 for each eye, are believed to give rise to all of the photoreceptors in the mouse (Mintz and Sanyal, 1970; Mintz, 1974). When earlier authors performed lineage analyses of PCs, however, they were unable to find evidence of clonal development. Mullen (1977) found an average clone size of 1 , while Oster-Granite and Gearhart (1981) calculated an average clone size of 2 to 4 cells. In both cases, the clone size is rather small. These investigators looked at spatially contiguous patches in the adult chimeras. Instead of contiguous patches, our procedure analyzes numerical units, each of which is distributed throughout the cerebellum. Our results suggest that clonal development is integral to cerebellar development in a numerical, but not a spatial, sense. Together, both types of analyses indicate that, during development, extensive mixing of the cells of the PC population occurs.

Our data can be used to estimate the time of commitment of the PCs. For 1 cell to give rise to 10,200 cells (or for 8 cells to give rise to 81,600 ), a minimum of 13.3 cell divisions are required. The average cell cycle time in mouse neural tube is estimated to be 7 to $10 \mathrm{hr}$ (Atlas and Bond, 1965; Kauffman, 1968; Wilson, 1974, 1981). Since PCs undergo their last cell division between embryonic days 11 and 14 (Miale and Sidman, 1961), the commitment event occurs between embryonic days 7.0 and 8.5. In the mouse, this is the neural plate to neural groove stage of development. This coincides with the time of determination of the nervous system as established by the work of the classical embryologists (discussed below).

Many different factors control the final number of cells in a neuronal population. The overproduction of neurons followed by a period of naturally occurring cell death has been described in many neural systems (Cowan, 1973; Purves and Lichtman, 1980). Although it has never been documented, it is possible that PCs also are overproduced and that the excess cells are removed by cell death. Instead of degenerating, the excess cells also might differentiate into some other cell type. Recall that we do not know if the progenitor cells also give rise to cell types in addition to PCs. If any of these processes were occurring, the total number of progenitors would not be affected, but more cells would have to be produced by each progenitor. Thus, additional cell divisions would be required, and therefore, 13.3 must be considered a minimum estimate of the number of cell divisions. In addition, it would seem as though the processes mentioned above should have obscured the integral multiples of one clone which were seen in the chimeras and the wild type animals. Since we do observe integrals, one possibility is that these processes do not occur to a significant extent during PC development. It is more likely that these processes occur relatively late vis à vis the commitment event and affect a large, well mixed population, and therefore, the proportion of cells affected is identical among the descendants of each of the progenitors.

The classical embryologists defined determination experimentally. A group of cells is said to be determined if, after transplantation or rotation, these cells continue to develop autonomously. For example, rotation of part of the neural ectoderm has no efiect before determination occurs. Only a few hours later, however, the rotated piece retains its original orientation and develops into structures which are rotated with respect to the rest of the CNS (Jacobson, 1964 in Kallen, 1965). Experiments in which portions of the neural plate are transplanted to ectopic sites confirm these results (Spemann, 1938; Kallen, 1958; Jacobson, 1959). Due to the inaccessibility of the mammalian embryo, our PC progenitors cannot be tested in this way. However, as discussed above, there is evidence for cell mixing during and after the neural plate stage (see also Jacobson and Hirose, 1981). Under these 
circumstances, the fate of the PC progenitors and their descendants cannot be flexible. If the fate of these cells was flexible and could be influenced by neighboring cells, then the loss of these cells' descendants by mixing would have resulted in a decreased number of PCs, and this number of PCs would not have been an integral multiple of the number in $\chi 11$. Thus, it seems that the event which we have been calling commitment is similar to classical determination since both involve the restriction of cell fate. The transplantation and rotation experiments, performed in chick and amphibia, were limited by the size of neural tissue which could be manipulated. Even the smallest pieces probably included many types of cells. On the other hand, these experiments reveal very accurately the time at which determination occurs. In our study, because of the estimates of cell cycle time and the number of divisions, we can only estimate the time of commitment. However, our analysis does accurately reveal the number of progenitors involved in commitment. Thus, these two approaches are complementary, and together, they indicate that on the order of 8 to 11 progenitors are committed to forming $\mathrm{PCs}$ during the neural plate stage of mouse development.

This report has expanded our understanding of neuronal differentiation. Our analysis has shown that a small number of progenitors gives rise to the entire population of PCs in the wild type mouse. These progenitors are selected early in development, approximately at the late neural plate stage. Thus, the fate of these neuroblasts are determined significantly before the last cell divisions. In addition, the descendants of these progenitors contribute to all functional subdivisions of the cerebellum.

\section{References}

Atlas, M., and V. P. Bond (1965) The cell generation cycle of the eleven-day mouse embryo. J. Cell Biol. 26: 19-24.

Caddy, K. W. T., and T. J. Biscoe (1979) Structural and quantitative studies on the normal $\mathrm{C} 3 \mathrm{H}$ and lurcher mutant mouse. Philos. Trans. R. Soc. Lond. (Biol.) 287: 167-201.

Cowan, W. M. (1973) Neuronal death as a regulative mechanism in the control of cell number in the nervous system. In Development and Aging in the Nervous System, M. Rockstein, ed., pp. 19-41, Academic Press, New York.

Dewey, M. J., A. G. Gervais, and B. Mintz (1976) Brain and ganglion development from two genotypic classes of cells in allophenic mice. Dev. Biol. 50: 68-81.

Falconer, D. S., and P. J. Avery (1978) Variability of chimeras and mosaics. J. Embryol. Exp. Morphol. 43: 195-219.

Hendry, I. A. (1976) A method to correct adequately for the change in neuronal size when estimating neuronal numbers after nerve growth factor treatment. J. Neurocytol. 5: 337-349.

Jacobson, C. -O. (1959) The localization of the presumptive cerebral regions in the neural plate of the axolotl larva. J. Embryol. Exp. Morphol. 7: 1-21.

Jacobson, C. -O. (1964) Motor nuclei, cranial nerve roots, and fibre pattern in the medulla oblongata after reversal experiments on the neural plate of axolotl larvae. 1. Bilateral operations. 7ool. Bidrag. Uppsala 36: 73-160.

Jacobson, M., and G. Hirose (1981) Clonal organization of the central nervous system of the frog. II. Clones stemming from individual blastomeres of the 32- and 64-cell stages. J. Neurosci. 1: 271-284.
Kallen, B. (1958) Studies on the differentiation capacity of neural epithelium cells in chick embryos. Z. Zellforsch Mikrosk. Anat. Abt. Histochem. 47: 469-480.

Kallen, B. (1965) Early morphogenesis and pattern formation in the central nervous system. In Organogenesis, $\mathrm{R} . \mathrm{H}$. Dittan and H. Ursprung, eds., pp. 107-128, Holt, Rinehart and Winston, New York.

Kauffman, S. L. (1968) Lengthening of the generation cycle during differentiation of the mouse neural tube. Exp. Cell Res. 49: 420-424.

Konigsmark, B. W. (1970) Methods for the counting of neurons In Contemporary Research Methods in Neuroanatomy, W. J. H. Nauta and S. O. E. Ebbesson, eds., pp. 315-339, Springer-Verlag, New York.

LeDouarin, N. M. (1980) The ontogeny of the neural crest in avian embryo chimeras. Nature 286: 663-669.

Levilt, P., M. L. Cooper, and P. Rakic (1981) Coexistence of neuronal and glial precursor cells in the cerebral ventricular zone of the fetal monkey: An ultrastructural immunoperoxidase analysis. J. Neurosci. 1: 27-39.

McLaren, A. (1976) Mammalian Chimeras, Cambridge University Press, London.

Miale, I. L., and R. L. Sidman (1961) An autoradiographic analysis of histogenesis in the mouse cerebellum. Exp. Neurol. 4: 277-296.

Mintz, B. (1962) Formation of genotypically mosaic mouse embryos. Am. Zool. 2: 432 .

Mintz, B. (1965) Genetic mosaicism in adult mice of quadriparental lineage. Science 148: 1232-1233.

Mintz, B. (1974) Gene control of mammalian differentiation. Annu. Rev. Genet. 8: 411-470.

Mintz, B., and S. Sanyal (1970) Clonal origin of the mouse visual retina mapped from genetically mosaic eyes. Genetics 64: $\mathrm{s} 43-\mathrm{s} 44$

Mullen, R. J. (1977) Site of pcd gene action and Purkinje cell mosaicism in cerebella of chimeric mice. Nature 270: 245-247.

Mullen, R. J., and K. Herrup (1979) Chimeric analysis of mouse cerebellar mutants. In Neurogenetics: Genetic Approaches to the Nervous System, X. O. Breakefield, ed., pp. 173-196, Elsevier/North-Holland, New York.

Mullen, R. J., and W. K. Whitten (1971) Relationship of genotypes and degree of chimerism in coat color to sex ratios and gametogenesis in chimeric mice. J. Exp. Zool. 178: 165-176.

Oster-Granite, M. L., and J. Gearhart (1981) Cell lineage analysis of cerebellar Purkinje cells in mouse chimeras. Dev. Biol. 85: 199-208.

Phillips, R. J. S. (1960) "Lurcher," a new gene in linkage group XI of the house mouse. J. Genet. 57: 35-42.

Purves, D., and J. W. Lichtman (1980) Elimination of synapses in the developing nervous system. Science 210: 153-157.

Spemann, H. (1938) Embryonic Development and Induction, reprinted by Hafner Publishing Co., New York.

Tarkowski, A. K. (1961) Mouse chimeras developed from fused eggs. Nature 230: 333-334.

Wenger, B. S. (1951) Determination of structural patterns in the spinal cord of the chick embryo studied by transplantations between brachial and adjacent levels. J. Exp. Zool. 116: 123-163.

Wetts, R., and K. Herrup (1982) Interaction of granule, Purkinjc, and inferior olivary neurons in lurcher chimeric mice. I. Qualitative studies. J. Embryol. Exp. Morphol. 68: 87-98.

Wilson, D. B. (1974) The cell cycle of ventricular cells in the overgrown optic tectum. Brain Res. 69: 41-48.

Wilson, D. B. (1981) The cell cycle during closure of the neural folds in the C57BL mouse. Dev. Brain Res. 2: 420-424. 